\title{
EL ALBA-TCP: de la emergencia a la resistencia
}

\author{
The ALBA-TCP: from emergency to resistance
}

ALBA-TCP: do surgimento à resistência

\section{María de Carmen Pérez González*}

\section{RESUMEN}

La integración centrada en los nexos económicos ocupa a los investigadores, especialmente la ocurrida en América Latina, cuyas particularidades han enriquecido la teoría. Desde su emergencia, la Alianza Bolivariana Para los Pueblos de Nuestra América-Tratado de Comercio de los Pueblos (ALBA-TCP) atrajo la atención de los estudiosos en un contexto propicio para su expansión, el cual fue sustituido por otro no favorable que la sitúa en una etapa caracterizada por la pérdida de miembros y cierto retroceso en algunos de sus proyectos, de ahí la noción de la existencia de dos etapas en su evolución. A pesar de su repliegue, el ALBA-TCP celebra sus cumbres, mantiene su carácter contrahegemónico, y crea una estrategia regional pos-COVID-19, lo cual sugiere una situación de resistencia. El presente artículo tiene como objetivo examinar, mediante la interpretación sociohistórica de la hermenéutica profunda de John B. Thompson, cómo se presenta la resistencia en el ALBA-TCP, de donde pueden derivarse enseñanzas para otros esquemas de ese orden en escenarios adversos.

\section{ABSTRACT}

Integration focused on economic links occupies researchers, especially in Latin America, whose particularities have enriched the theory. Since its emergence, the Bolivarian Alliance for the Peoples of Our America-People's Trade Agreement (ALBA-TCP)
Palabras clave:

ALBA-TCP, emergencia, integración económica regional, resistencia.

Keywords: ALBATCP, emergence, regional economic integration, resistance.

\footnotetext{
* $\quad$ Magíster en Temas Interdisciplinarios de América Latina, el Caribe y Cuba, por la Universidad de la Habana, Cuba. Universidad de la Habana, Cuba. E-mail: maracose@ nauta.cu
} 
attracted scholars' attention in a context conducive to its expansion. Replaced unfavorably and set on a stage characterized by the loss of members and project setbacks. Hence, the notion of the existence of two stages in its evolution.

Despite its withdrawal, the ALBA-TCP celebrates its summits, maintains its counter-hegemonic character, and creates a post-COVID-19 regional strategy, suggesting a resistance situation. Through the socio-historical interpretation of John B. Thompson's profound hermeneutics, the present article examines how ALBA-TCP exhibits resistance and what it teaches us for other similar systems in adverse scenarios.

\section{RESUMO}

A integração enfocada nos vínculos econômicos ocupa os pes-

Palavras-chave: quisadores, especialmente a ocorrida na América Latina, cujas particularidades enriqueceram a teoria. Desde seu surgimento, ALBA-TCP, surgimento, a Aliança Bolivariana para os Povos da Nossa América - Tratado de Comércio dos Povos (ALBA-TCP) atraiu a atenção dos estuintegração econômica diosos em um contexto propício para sua expansão, o qual foi regional, substituído por outro desfavorável que a coloca em uma etapa resistência. caracterizada pela perda de membros e certo retrocesso em alguns de seus projetos. Daí a noção da existência de duas etapas em sua evolução. Apesar de seu recuo, a ALBA-TCP celebra suas reuniões, mantém seu caráter contra-hegemônico e cria uma estratégia regional pós-COVID-19, o que sugere uma situação de resistência. O presente artigo visa examinar, através da interpretação sócio-histórica da hermenêutica profunda de John B. Thompson, como se apresenta a resistência na ALBA-TCP, da qual podem ser extraídas lições para outros esquemas dessa ordem em cenários adversos. 


\section{Introducción: la integración en tela de juicio}

Si bien al inicio del siglo XXI dominó un contexto que favoreció la constitución y el fortalecimiento de asociaciones integracionistas en América Latina (Briceño, 2014), es perceptible una modificación de tal contexto en la segunda década que no la beneficia (Silva, Noyola y Kan, 2017). El regionalismo latinoamericano manifiesta rasgos de una nueva reconfiguración que rescata los vínculos con Estados Unidos, comulga con el desmontaje de la intervención pública y abandona la dimensión social (Aponte, 2018). Empero, la Alianza Bolivariana Para los Pueblos de Nuestra América-Tratado de Comercio de los Pueblos (en adelante ALBA-TCP) continúa siendo reconocida por su proyección contrahegemónica (Aponte, 2018; Muhr, 2010).

El pernicioso contexto global y regional adopta manifestaciones en las instancias integracionistas tales como la retirada de algunos de sus miembros, el abandono de proyectos y el debilitamiento de los esquemas. De ese fenómeno no ha estado ajena la Unión Europea, la más avanzada forma de integración y referente teórico y práctico.

En el ALBA-TCP esa situación se proyecta con particular nocividad, debido a la hostilidad contra algunos de sus miembros. El desmembramiento y debilitamiento de sus proyectos caracterizan la actual etapa por la cual está transitando esa instancia regional. Igualmente dañina es la situación interna venezolana, dado el rol que ese país ha desempeñado en el financiamiento de los proyectos grannacionales a partir de los ingresos derivados de la producción petrolera (Gratius y Puente, 2018).

A partir de la fundación del ALBA-TCP en el año 2004, la discontinuidad que se aprecia en su evolución permite establecer dos etapas diferenciables: una de ascenso y otra de estancamiento. La primera transcurrió durante sus años iniciales, cuando el ALBA-TCP se convirtió en centro de atención de las fuerzas de izquierda, al ver en ella el resurgir de la utopía frustrada con la caída del campo socialista que removió las bases teóricas e ideológicas de los proyectos emancipatorios. También lo fue en la academia, toda vez que la asociación regional propuesta, no encontraba explicación en la teoría aceptada y presentó el reto de elucidar sus fundamentos teóricos. La segunda etapa coincide con la segunda década de existencia del ALBA-TCP y, aunque 
esa organización continúa atrayendo la atención de especialistas y movimientos sociales, su evolución no acusa el dinamismo de aquella. En la producción teórica es igualmente perceptible una diferenciación en el acercamiento de los autores al ALBA-TCP.

En efecto, los numerosos estudios en torno al ALBA-TCP inicialmente han girado en torno a sus valores, con énfasis en sus propuestas novedosas a través de las cuales se distancia de la práctica de las demás instancias integracionistas. También se detienen en elementos considerados obstáculos para un mayor despliegue de sus proyectos. No obstante, en ambas interpretaciones es reconocida como una modalidad estimable en el regionalismo latinoamericano, en tanto alternativa a la dependencia, subdesarrollo y dominación. En ese sentido, despiertan especial interés las relaciones entre sus miembros, principalmente la solidaridad y la complementariedad (Borón, 2008). Particular notoriedad posee para los estudiosos, la naturaleza de las relaciones promovidas por esa asociación, que para el comercio internacional propone la colaboración y la cooperación, en términos de ventajas cooperativas (Angelilli, 2016).

La presencia de los elementos histórico e ideológico, así como su carácter contrahegemónico (Muhr, 2010), también se hallan entre las perspectivas empleadas por los autores en su acercamiento al ALBATCP. Al respecto, forman parte de los acercamientos de los especialistas al ALBA-TCP la creación de una conciencia colectiva regional en correspondencia con la dimensión regional del proyecto, que retoma en sus bases ideológicas el pensamiento latinoamericano bolivariano (Cole, 2010); su relación con el regionalismo (Aponte, 2014); y el reconocimiento de una integración de nuevo tipo (Stédile, Piñero y Bertoldi, 2013). La débil institucionalidad, así como la ausencia del carácter supranacional en el marco jurídico (Soto y Morales, 2014) del ALBATCP, también cuentan en los análisis de los autores, quienes advierten el riesgo contenido en esos elementos - tanto en el cumplimiento como en la continuidad en el tiempo-, de los compromisos políticos entre los estados miembro.

Al transitar hacia la segunda etapa, los estudios se detienen en los elementos que obstaculizan la integración en general y esa asociación en particular. Algunos autores evalúan la actual etapa por la que está atravesando la asociación como de estancamiento o de crisis existen- 
cial. En la primera denominación el agotamiento del regionalismo latinoamericano de principios del siglo XXI se atribuye como condicionante, ya que ello privilegió la concertación política intergubernamental, tomó en cuenta la necesidad de una participación del Estado en la dirección de la economía nacional, y potenció la protección social (Silva et al., 2017); mientras la segunda, es derivada por Gratius y Puente (2018) de un análisis comparado con la Alianza del Pacífico.

Sin embargo, el ALBA-TCP continúa siendo reconocida como una propuesta emanada de los países dominados (Angelilli, 2016; Aponte, 2018; Fermín, 2009) que, no obstante la adversidad del entorno, mantiene la concertación, celebra sus cónclaves y aunque debilitados, sus proyectos prevalecen. En medio de la pandemia COVID-19, cuando se enseñorea el desconcierto y evidencian sus limitaciones los sistemas de salud como parte de las estructuras de gobierno, el ALBA-TCP se moviliza para enfrentarla y elaborar una estrategia pos-COVID-19.

Por esas razones, se estimó oportuno acudir a los estudios teóricos de las prácticas de resistencia, por considerarla afines con la actuación del ALBA-TCP, que aún en circunstancias adversas mantiene sus proyectos y preserva sus valores fundacionales. Tal análisis podría aportar enseñanzas de valor para entidades integracionistas latinoamericanas, igualmente impactadas por la nocividad del entorno.

En la realización del presente estudio se acudió a la metodología analítico-hermenéutica profunda de Thompson (1998), al analizar cómo el ALBA-TCP responde al desfavorable contexto global y regional, desde la perspectiva de las prácticas de la resistencia (Giroux, 1983; Guerrero, 2005). Comprende un enfoque sociohistórico que toma en cuenta la evolución de esa asociación en su contexto, con lo cual permite establecer dos períodos diferenciables mediante una comparación: uno de avance y otro de estancamiento.

\section{El ALBA-TCP en un contexto adverso}

La evolución de la propuesta integracionista latinoamericana y caribeña ALBA-TCP, constituida en el año 2004, manifiesta a partir de su segunda década diferencias en relación con su comportamiento durante su primer decenio. En sus primeros 12 a 15 años, esa organización estuvo caracterizada por su expansión, expresada en la adhesión de 
miembros y la promoción de proyectos. Con posterioridad, a pesar de que se aprecia la retirada de algunos de sus miembros y el detenimiento de ciertos de sus proyectos, la organización conservó su estructura y funcionamiento. De ahí el interés en una reflexión en torno al ALBATCP tomando como referente las teorías de la resistencia.

Mientras algunos esquemas abandonan sus postulados primigenios (Silva et al., 2017), la asociación bolivariana continuó defendiendo sus valores fundacionales. Tal comportamiento sugiere una modalidad de resistencia colectiva, que actúa en el escenario internacional y cuyo centro se halla en la integración económica regional. Es por esas razones que el presente artículo está guiado por el objetivo de examinar el ALBA-TCP, utilizando como referentes los constructos teóricos asociados a las prácticas de la resistencia. Para ello, se apela aquí al empleo de la hermenéutica con la intención de contrastar los postulados teóricos de la resistencia con la dinámica de la organización regional objeto de estudio.

Como los estudios de la resistencia están asociados con grupos sociales que sufren algún tipo de exclusión, así como con los actores del proceso de enseñanza aprendizaje, para interpretar este fenómeno en relación con el ALBA-TCP se estimó necesario acudir a la hermenéutica profunda que propone John B. Thompson (1998) para las ciencias sociales. El análisis sociohistórico, el análisis formal o discursivo, y la interpretación/reinterpretación, aparecen integrados en su propuesta como procedimientos dentro de un marco metodológico general, el cual permite hacer uso de uno u otro procedimiento, en razón del objeto de estudio. En este caso, por tratarse de una asociación regional integracionista, el análisis sociohistórico resulta pertinente, teniendo en cuenta que el contexto, los actores, las instituciones y los simbolismos del ALBA-TCP, aportan en la elucidación de sus prácticas de resistencia.

Para el proceso de investigación se empleó la revisión bibliográfica y documental que permitió la interpretación de textos científicos, discursos y documentos, así como la reinterpretación de las teorías de la resistencia en la ejecutoria del ALBA-TCP en el último lustro. Con el empleo de la fase de análisis sociohistórico de la hermenéutica profunda de Thompson (1998), se pudo describir el contexto en el que surgió y evolucionó el ALBA-TCP como organización, en el marco del proceso integracionista latinoamericano. 
A partir de ese análisis se reconoció una modificación en la dinámica de esa instancia que permite establecer dos etapas desde su constitución hasta la actualidad. Los elementos identificados en el establecimiento de las dos etapas están asociados con la incorporación de naciones al ALBA-TCP y la implementación de proyectos. En la primera de las etapas la curva es ascendente, mientras que luego es descendente.

La interpretación/reinterpretación de la teoría de la resistencia, parte de la noción de que el nivel regional es propicio para la ocurrencia de prácticas de resistencia. En el continente latinoamericano, la dependencia como forma de ejercicio del poder desde una nación extranjera, genera expresiones tanto en la teoría como en la práctica. En ese sentido, el surgimiento del ALBA-TCP puede considerarse una respuesta de un grupo de naciones desde la resistencia. De mayor relevancia resulta la ejecutoria más reciente del ALBA-TCP, especialmente después de 2015, como una práctica de resistencia de una instancia regional.

La resistencia como término se halla asociado con las dimensiones físicas y sociales. Esta última es la que resulta de interés en el presente estudio, cuya génesis se remonta a la década del 60 , resultado de las interpretaciones de Michel Foucault en torno al poder, cuando expone que lejos de estar concentrado, irradia en múltiples sentidos y acusa una tendencia al autoritarismo, pero simultáneamente propicia la generación de prácticas de resistencia (Hidalgo, 2014). Contrariamente al sentido de la resistencia física, la social supone una proyección derivada del análisis crítico de la realidad. El centro de su atención estuvo en las modalidades en que los movimientos sociales se oponían al estado de cosas existente y socialmente aceptado. Se trata, entonces, de una resistencia que no es pasiva, por el contrario: de ella emana una propuesta de transformación.

Posteriormente, otros autores realizaron estudios teóricos de las prácticas de resistencia desde diversas perspectivas, las que incluyen los conflictos sociales y la relación dominantes-dominados (Vargas, 2014), la educación específicamente en la corriente de la pedagogía crítica (Guerrero, 2005), en las manifestaciones culturales (Sánchez, 2013), y en la comunidad (González, Colmenares y Ramírez, 2011). Considerada como una respuesta a un currículo autoritario que mues- 
tra un proyecto alternativo en ciernes (Giroux, 1983), la resistencia adopta diversas modalidades. A través de esas formas, desafían la imposición ideológica de las clases dominantes, al revelarse como expresiones contrahegemónicas que pretenden la reproducción ininterrumpida de las relaciones sociales de producción inherentes. Consecuentemente, los portadores de esas expresiones dejan de ser simples agentes acríticos de la reproducción social, para convertirse en actores con una visión cuestionadora de la realidad.

La hegemonía no solo ha sido identificada con el mantenimiento forzoso de la dominación, sino que se apoya en las prácticas y estructuras sociales asumidas. Entre las primeras se hallan las normas observadas por los miembros de la sociedad en el mantenimiento del orden social y las costumbres socialmente aceptadas. Mientras en las segundas, actúan las instituciones tales como la familia, la escuela, las entidades religiosas, el Estado, el sistema político y los medios de comunicación, que han ganado espacio asociados al desarrollo de internet y las redes en línea. Tanto las prácticas como las estructuras sociales contribuyen a reproducir y perpetuar las relaciones dominantes. Justamente, la resistencia está dirigida contra las acciones de la cultura dominante.

Mediante las teorías de la resistencia es posible interpretar la etapa por la que transita el ALBA-TCP, caracterizada por la pérdida de miembros y cierto retroceso en algunos de sus proyectos, por una parte, mientras que por otra, ha mantenido su funcionamiento, carácter contrahegemónico y prepara una respuesta colectiva regional ante la crisis ocasionada por el COVID-19. Ello es muestra de la vitalidad de la organización, al tiempo que permite inferir que proyecta nuevas propuestas frente a las prácticas dominantes en la integración económica regional.

\section{El ALBA-TCP en el regionalismo latinoamericano del siglo XXI}

Desde su surgimiento en el año 2004, el ALBA-TCP se convirtió en centro de atención de los estudiosos de la integración. La propuesta de Hugo Chávez de la Alternativa Bolivariana para las Américas, en diciembre de 2001 durante la celebración de la Cumbre de Jefes de Estado de la Asociación de Estados del Caribe - cuando EE.UU. ultimaba la constitución del Acuerdo de Libre Comercio para las Américas 
(ALCA)—, le otorgó un carácter contrahegemónico (Muhr, 2010). Las expresiones de rechazo de los pueblos latinoamericanos a la propuesta norteamericana, conquistaron la Academia.

En los debates que sucedieron a la proclamación del ALCA, pueden ser identificadas dos aristas: una se dedicó a fundamentar su esencia como un nuevo mecanismo de dominación y a predecir las negativas consecuencias que tendría ese proyecto para América Latina; mientras la otra, emergió en 2004 como un proyecto alternativo, cuando Hugo Chávez propuso la creación del ALBA y junto a Fidel Castro, se convirtieron en sus fundadores. La distinguen de otras entidades del mismo orden sus propósitos, las relaciones que promueve, el criterio de desarrollo y la prioridad otorgada a la atención social (Pérez, 2016).

Cuando surge el ALBA-TCP, la integración había recorrido más de 50 años en América Latina y el Caribe. La adhesión a la novedosa propuesta en esa área estuvo alentada por la industrialización para la sustitución de importaciones. Posteriormente, adicionó la reducción de las desigualdades y, más tarde, un estilo de desarrollo centrado en el ser humano, criterios presentes en el ALBA-TCP.

El primero de los esquemas data del año 1951 cuando El Salvador, Guatemala, Honduras, Nicaragua y Costa Rica, constituyeron la Organización de Estados Centroamericanos (ODECA). En 1960 adoptó la denominación de Mercado Común Centroamericano (Mercomun) hasta 1991, cuando se tituló Sistema de Integración Centroamericana (SICA). La institucionalidad lograda en esa asociación está considerada como su rasgo más distintivo, comparable con la Unión Europea (Samuha, 2015).

Representante del viejo regionalismo (Samuha, 2015), la Asociación Latinoamericana de Libre Comercio (ALALC), gestada en 1959 ${ }^{1}$ fue constituida en 1960 por Argentina, Brasil, Chile, México, Paraguay y Uruguay. En 1980, se transformó en la Asociación Latinoamericana de integración, ALADI.

1 La solicitud de adhesión presentada por Cuba no fue aceptada, alegando la diferencia de sistema sociopolítico. Ello confirma el carácter sistémico que ha caracterizado a la integración económica regional del siglo XX. 
A estas instancias les sucedieron el Pacto Andino (1969), que derivó en Comunidad Andina de Naciones; la Comunidad del Caribe, Caricom (1973); el Mercado Común del Sur, Mercosur (1991); el Sistema de Integración Centroamericana, SICA (1991); y la Alternativa Bolivariana para los pueblos de América (2004), que posteriormente se une al Tratado de Comercio de los Pueblos propuesto por Bolivia (2006) y adopta su actual denominación -ALBA-TCP_; la Unión de Naciones del Sur, Unasur (2008); la Comunidad de Estados Caribeños y Latinoamericanos, Celac (2010) y Alianza Pacífico, AP (2012).

La práctica de la integración en América Latina ha estado acompañada de las reflexiones teóricas que, si bien se remiten a la reconocida como clásica, se detiene en su realidad signada por el subdesarrollo y la dependencia. Entre los años 60 y 70 del siglo XX, se desarrollaron teorías asociadas con la interpretación de la situación latinoamericana (Bárcena, 2015; Briceño, 2014). Dos corrientes de pensamiento centraron la atención en torno a las relaciones establecidas por los países dominantes del sistema capitalista, con énfasis en el intercambio desigual y la dependencia. Esas perspectivas se distanciaron del liberalismo, en tanto que sus análisis del comercio internacional y las relaciones derivadas revelaron inequidades en su instrumentación por parte de las empresas trasnacionales.

Es por ello que la primera etapa de la integración en América Latina (años 60 y 70 del pasado siglo) si bien estuvo influenciada por el pensamiento clásico, la corriente estructuralista - representada por Raúl Prebisch, Celso Furtado y Ruy Mauro Marini- así como la teoría de la dependencia - Tehotonio Dos Santos y Osvaldo Sunkel- fueron dominantes. De sus análisis surgió la propuesta de un modelo asentado en la industrialización y la sustitución de importaciones (ISI) (Bárcena, 2015).

Ambas líneas de pensamiento tuvieron en común un escenario propicio para la generación de interpretaciones que, sin desconocer la herencia teórica, se correspondieran con el contexto latinoamericano. Tal escenario fue la Comisión CEPAL y, por ello, la integración llevó el sello del estructuralismo y la teoría de la dependencia (Bárcena, 2015).

En sus inicios, el pensamiento en torno a la integración en el seno de la CEPAL se pronunció por la industrialización para sustituir 
importaciones. Posteriormente, adicionó la reducción de las desigualdades (Briceño, 2014) y más tarde —década de 1970 - se reorientó hacia los estilos de desarrollo (Bárcena, 2015). Los temas macroeconómicos cobraron importancia en la década del 80, en tanto que en los 90 se pronunció por la transformación productiva con equidad. En el tránsito del siglo XX al XXI, las ideas neoestructuralistas de Fernando Fajnzylber y José Antonio Ocampo pusieron de relieve las asimetrías, la desfavorable inserción internacional, la vulnerabilidad externa y los ciclos económicos.

Por su parte, en el escenario latinoamericano es posible apreciar diferencias en los esquemas actuantes, que guardan relación con los principios de los países integrantes en sus relaciones internacionales, lo cual se refleja a nivel regional en la incorporación de esquemas congruentes con los propósitos de los países. Los especialistas los agrupan en dos tendencias: una de ellas se afilia al mantenimiento de los postulados del libre comercio, la expansión de las inversiones y la inserción en el nivel internacional, lo que se expresó en la suscripción de acuerdos bilaterales con EE.UU. en los casos de México, Chile, Colombia, Perú y varios países centroamericanos; en tanto que en el nivel regional, la tendencia fue la adhesión a esquemas más afines a la concertación política como Unasur.

Simultáneamente, la otra tendencia estuvo animada por la implementación de procesos de integración que relativizaran el comercio y otorgaran prioridad a la agenda social. Este es el caso de Bolivia, Cuba, Ecuador, Venezuela, Nicaragua -miembros del ALBA-TCP- y Brasil y Argentina, entre otros (Montenegro-Braz, 2017). En analogía con la teoría convencional, el ALBA-TCP - al igual que la Unasur y la CELAC-, no siguen las pautas usuales de las instancias integracionistas reconocidas, sino esquemas o procesos de cooperación, colaboración y concertación (Angelilli, 2016). No obstante, el estudio de estas asociaciones, junto con las demás experiencias latinoamericanas, enriquece la teoría de la integración especialmente por aportar características novedosas. La emergencia de esas tres asociaciones con propuestas distanciadas de la teoría y la práctica de la integración tradicional, es lo que ha conducido a identificarlas como promotoras de un nuevo regionalismo estratégico (Aponte, 2014). 
La práctica de la integración latinoamericana condujo a Chávez a considerar la necesidad del protagonismo de los países latinoamericanos en la identificación de sus aspiraciones. En un primer momento, el proceso de integración en América Latina estuvo orientado hacia el mercado internacional mediante la sustitución de importaciones (Bárcena, 2015), cuyo fundamento se nutrió de las teorías desarrolladas en el seno de la CEPAL. Posteriormente, el neoliberalismo en su expansión, alcanzó las propuestas integracionistas, introduciendo la desregulación del comercio (Bárcena, 2015). Esas experiencias pueden haber aportado elementos a Chávez en la formulación de una propuesta de integración distanciada de las precedentes.

Igualmente, Chávez distinguió dos niveles en la integración: el interno y el externo (Pérez, 2016). En el primero consideró la desintegración resultante de la penetración extranjera y las elites nacionales. En el segundo situó, justamente, el escenario en que proyectaba el ALBA: América Latina y el Caribe. Lo político, lo social, tanto como lo ideológico y lo económico, los identificó como dimensiones en su propuesta (Pérez, 2016). Así mismo, concibió la ideológica en las luchas libertarias y el pensamiento liberador de ella derivado, enriquecido por la historia de los pueblos durante el período republicano, acuñado también por otros autores (Angelilli, 2016). En la participación social sin exclusiones sustentó la política, la construcción desde abajo aportaba a la integración en el ALBA la especificidad reconocida por teóricos y hacedores de políticas (Benzi, 2014).

Conocedor del sobredimensionamiento otorgado al comercio en la práctica, usualmente identificado con la dimensión económica, Chávez lo estimó en un lugar de menor relevancia. Empero, les otorgó a la cooperación y la complementación una notabilidad que las elevaron al rango de principios del ALBA.

Fue por medio de una declaración política firmada por los jefes de Estado Fidel Castro y Hugo Chávez, en diciembre de 2004 en La Habana, que fue constituida la Alternativa Bolivariana para los pueblos de América. Ese hecho guarda relación con las teorías de la resistencia, por tratarse de una propuesta que cuestionaba el orden existente y proponía otros principios de relacionamiento. Desde ese momento, la emergente asociación regional comenzó un proceso de ampliación reflejado tanto en la incorporación de miembros, como en los proyectos 
de colaboración, especialmente de carácter social. Los países que se incorporaron luego suscribieron la declaración primigenia.

Entre 2004 y 2014 transcurrió la primera década del ALBA, durante la cual se produjo la adhesión de varios países a la nueva propuesta integracionista. En 2006 lo hizo Bolivia² y propuso el Tratado de Comercio de los Pueblos, con lo cual la asociación adoptó su actual denominación: ALBA-TCP. También lo hicieron Nicaragua (2007), Hondu$\operatorname{ras}^{3}$ y Dominica (2008), Ecuador ${ }^{4}$, Antigua y Barbuda, San Vicente y las Granadinas (2009), Surinam (2012), Santa Lucía ${ }^{5}$ (2013), San Cristóbal y Nieves y Granada (2014). Haití es invitado especial. Al afiliarse a la organización regional, estos países aceptaron las bases de la declaración constitutiva de 2004. De esa forma, la institucionalidad funcionaba sobre la base del consenso.

Como se mencionó, el ALBA-TCP otorgaba prioridad a la dimensión social del desarrollo (Aranda, 2015), de ahí la promoción de proyectos sociales entre sus miembros, los cuales se concretaron en programas de amplio alcance dirigidos a revertir la desatención de la población de menores ingresos, especialmente en las esferas de la salud y la educación. Destacan en ese sentido los programas de Alfabetización ${ }^{6}$, la Operación Milagro ${ }^{7}$ y la Atención a discapacitados ${ }^{8}$.

Los programas sociales mencionados fueron financiados con el Fondo ALBA-Caribe, derivado del acuerdo de cooperación Petrocaribe y contaron con el aporte tecnológico de Cuba. La experiencia de la alfabetización ha sido extendida a países de África, Asia, Oceanía y Europa y forma parte de las proyecciones de Cuba y Venezuela a nivel del ALBA-TCP. También países no miembros del ALBA-TCP fueron be-

2 Tras el golpe de Estado, que depuso al presidente de Bolivia en noviembre de 2019, ese país se retiró del ALBA-TCP.

3 En 2009, luego de un golpe de Estado que provocó el derrocamiento del presidente de Honduras, el nuevo gobierno se retiró del ALBA-TCP.

4 En 2018, luego de las elecciones presidenciales en Ecuador, el nuevo gobierno decidió retirarse del ALBA-TCP.

5 Santa Lucía se retiró del ALBA-TCP en 2020.

6 Con el programa "Yo sí puedo" fueron alfabetizados 6 millones de personas.

7 La aplicación del programa "Operación Milagro" devolvió la visión a 2 millones de personas.

8 Derivado de un estudio multidisciplinario, fue diagnosticado y asistido 1 millón 280 mil personas. 
neficiados con atención oftalmológica y en la formación de médicos y licenciados en cultura física.

Al respecto, los estudiosos coinciden en la afirmación de que la dimensión social aporta el sello distintivo en relación con asociaciones del mismo orden y es también en ella donde se registran sus principales éxitos (Benzi, 2014). De ahí el interés mostrado por especialistas y políticos en el asunto y es por lo mismo que cualquier propuesta de desarrollo del ALBA-TCP no debe desestimarse.

Sin embargo, entre los rasgos comunes de los países afiliados al ALBA-TCP se cuentan el insuficiente crecimiento económico, la escasa variedad de producciones, la desfavorable inserción en el mercado internacional, insuficientes fuentes de financiamiento y el endeudamiento de sus economías, lo cual es relevante ya que, pese a estas características propias del subdesarrollo y la dependencia, pudieron fomentar políticas sociales en apoyo de la población menos favorecida.

Si bien los proyectos sociales resultan relevantes en el ALBA-TCP, esa instancia también desarrolló proyectos productivos, financieros y comerciales, los cuales fueron bautizados con término "grannacional" por Hugo Chávez, y que se organizaron en torno a objetivos estratégicos para la asociación en la que interviniera más de un país. Petrocaribe y el Sistema Unitario de Compensación Regional, SUCRE, destacan entre las grannacionales por incluir a todos los miembros del ALBATCP.

La grannacional Petrocaribe (2005) está considerado el instrumento de mayor amplitud como suministrador de petróleo y financista de programas, particularmente los sociales. Se identifica con las relaciones de cooperación que caracterizan la organización donde priman las ventajas cooperativas (Angelilli, 2016), las cuales se distancian de las tradicionales.

Petrocaribe comprende varios objetivos: el petróleo, el gas, la electricidad, la tecnología, la capacitación y las fuentes alternativas, aunque los propósitos de mayor impacto se concentran en el suministro de petróleo y gas en condiciones preferenciales. En ese proyecto participan otros países latinoamericanos, caribeños y de Estados Unidos, además de los miembros del ALBA-TCP. Sin embargo, a partir de 2015, la caída del precio de ese producto, unido a la situación interna vene- 
zolana, provocó una contracción en las posibilidades de reproducción de ese modelo.

Uno de los objetivos de la integración - a tenor de la evolución de la Unión Europea como su referente teórico-, es el empleo de una moneda común. Tanto el Consejo de Ayuda Mutua Económica, CAME, como la Unión Europea incluyeron las instituciones financieras en sus proyectos integracionistas respectivos. En América Latina, y siguiendo esas experiencias, existen tres mecanismos de sistemas de pagos internacionales:

- Convenio de Pagos y Créditos Recíprocos de la Asociación Latinoamericana de Integración, CPCR-ALADI.

- Sistema de Pagos en Monedas Locales entre Argentina y Brasil, SML.

- Sistema Unitario de Compensación Regional, SUCRE.

La constitución del SUCRE a nivel del ALBA-TCP se produjo en el año 2008 y comprendió los siguientes mecanismos: unidad de cuenta, Consejo Monetario Regional, Cámara Central de Compensación, Fondo de reservas y Convergencia Comercial, y el Banco del ALBA. La propuesta del SUCRE incluye una moneda virtual de igual denominación y cumple una función valorativa, aunque la de medio de circulación no le es propia, pues las transacciones son liquidadas con las monedas de los países miembro. La emisión y asignación de sucres se encuentra respaldada por títulos de valor o efectivo en moneda nacional, mientras la tasa de cambio se fija mediante una canasta de monedas.

En la práctica, los intercambios comerciales y la presencia del sucre en las transacciones no ha sido significativa, lo que evidencia que no se ha desplegado en toda su amplitud. No obstante, su utilidad para facilitar y estimular el comercio sin la intervención de las divisas (Benzi, 2014) como alternativa de los países miembro frente al dólar continúa latente (Chávez, 2005). Por ello, surgió la propuesta del Consejo Económico del ALBA-TCP de fortalecer el sucre, tanto la moneda como el banco al cual se adscribe, con lo cual se prevé que la propuesta del Petro habrá de desempeñar un importante rol (Granma, 2020).

A pesar de las dificultades de los países que permanecen asociados al ALBA-TCP, los programas emprendidos se mantienen aunque con 
limitaciones, donde los de carácter social siguen ocupando un lugar de preferencia. Especial atención han recibido la educación, la salud y el empleo, reflejadas en la reducción de la pobreza y las desigualdades generadas por las políticas distributivas precedentes. Ello se corresponde con las bases fundacionales de esa instancia integracionista que posee un carácter contrahegemónico entre las cuales cuentan sus propósitos, las relaciones que propone, la visión del desarrollo y la relevancia de la dimensión social.

En otro orden, pero igualmente relevante, se presenta el desfavorable contexto sociopolítico en que evolucionan los países líderes de ese esquema integracionista: Venezuela y Cuba, si bien otros países han sido objeto de presiones e intentos de desestabilización. Particularmente adverso fue para Bolivia y Ecuador, países donde las fuerzas populares perdieron el poder político. Estos cambios en el espacio de la izquierda en el continente latinoamericano han favorecido acciones contra los gobiernos populares, apoyadas por la actual administración de EE.UU.

\section{Una práctica regional de resistencia: el ALBA-TCP}

Si bien la emergencia del ALBA-TCP se vio acompañada por la incorporación de miembros y de proyectos de impacto especialmente de carácter social, algo muy diferente se produce a partir del año 2015, con la caída del precio del petróleo, el alza de los precios de las importaciones, la pérdida de poder político de la izquierda y la agresividad del gobierno norteamericano.

No obstante, la organización mantuvo algunos de sus proyectos y no ha dejado de celebrar sus cumbres ni de pronunciarse en los escenarios internacionales. Esos elementos sugieren una situación de resistencia que no es pasiva ni se halla desarticulada del contenido contrahegemónico originario del ALBA-TCP. De ahí el interés en la presente investigación de analizarla a partir de los referentes teóricos de la resistencia. Una mirada a la evolución más reciente en otros esquemas integracionistas también evidencia la retirada de algunos de sus miembros y el estancamiento de los proyectos, al igual que ocurre en el ALBA-TCP, pero a diferencia de este, no dan similares señales de vitalidad, al no presentar una propuesta que conserve sus bases fundacionales. 
A juicio de algunos autores, el contexto mundial y regional no favorece la integración (Gratius y Puente, 2018), ya que este no solo impacta negativamente en las asociaciones integracionistas, sino también repercute en los tratados de libre comercio, donde además del escenario económico, influye con particular fuerza el componente político. Al respecto, el reforzamiento de las posiciones proteccionistas del gobierno de Donald Trump, se proyecta contra los organismos de las Naciones Unidas con la negativa a aportar sus contribuciones e incluso con la amenaza de su retirada. El Tratado de Libre Comercio en el que participa ese país junto con Canadá y México se mantiene en un estancamiento debido a las exigencias de EE.UU, cuya administración decidió su retiro del Acuerdo de Asociación Transpacífico (TPP).

De igual modo, los procesos integracionistas asociados al regionalismo posliberal (Silva et al., 2017) se debilitan a partir de los cambios acaecidos en varios países latinoamericanos, donde los nuevos gobernantes relegaron los compromisos contraídos por sus antecesores con instancias de ese orden. La salida de Argentina, Brasil, Chile, Colombia, Perú y Paraguay de la Unasur en 2018, así como la suspensión de Venezuela del Mercosur en 2016, son reflejo de los procesos que están ocurriendo al interior de la integración en América Latina, los cuales provocan su debilitamiento.

Del mismo modo, en el Mercosur se aprecia la desfavorable tendencia de la integración. Aunque esa organización proyecta una reorientación, esta marca un giro en la dinámica recorrida desde sus inicios, ya que sus pasos apuntan en el sentido de la promoción de las relaciones con la Unión Europea y la Asociación de Naciones del Sudeste Asiático, ASEAN, en cuanto a instancias regionales, así como con Canadá y Corea del Sur en lo concerniente a países.

Esas manifestaciones de los procesos de integración en América Latina han conducido a los estudiosos a considerar una nueva reconfiguración en el regionalismo de principios del siglo XXI: el regionalismo estratégico (Aponte, 2018) que había dado lugar a la aparición de asociaciones menos propensas a los vínculos con EE.UU., y más proclives al reforzamiento con sus vecinos latinoamericanos, la intervención pública o estatal y la dimensión social, así como a la emergencia de una instancia contrahegemónica: el ALBA-TCP. Solo esta última se 
mantiene conectada a sus bases fundacionales en el mapa de la integración en América Latina, lo cual sugiere una actitud de resistencia.

Sin embargo, se trata de una situación que no es exclusiva de la región latinoamericana, ya que también se manifiesta en la Unión Europea, cuyo momento cumbre fue el protocolo inédito de retirada de Reino Unido concluido a principios de 2020. En este caso, fue necesario un largo proceso de transformaciones legales asociado a la supremacía de los instrumentos comunitarios sobre los nacionales y a la obligatoriedad que asumen los miembros por el cumplimiento de los compromisos.

Particular efecto provocan el contexto global y regional sobre el ALBA-TCP, en el cual la dimensión política resulta clave, toda vez que su marco jurídico no posee ascendencia sobre el nivel nacional de los países miembros, así como el rol preponderante de los jefes de Estado en la toma de decisiones de la asociación. Ello se refleja en la retirada de varios de sus miembros y en el detenimiento de algunos de sus proyectos. Esas razones han conducido a algunos autores a considerar que el ALBA-TCP se encuentra en un estancamiento (Silva et al., 2017), o bien, en una crisis existencial (Gratius y Puente, 2018).

$\mathrm{Al}$ respecto, ya desde el año 2008, la brusca caída de los precios del combustible se reflejó en la contracción de la economía venezolana. Sus efectos más visibles se proyectaron sobre la disponibilidad de recursos financieros que afectó los proyectos de la organización. Pero a partir del año 2015, a esos factores se le adicionaron la inestabilidad interna de Venezuela conducida por las fuerzas de oposición y el recrudecimiento de la agresividad del gobierno norteamericano en una tendencia ascendente. Estos dos últimos factores se han convertido en la principal amenaza al proyecto sociopolítico de la nación venezolana, en tanto que el descenso de los recursos financieros a partir de la renta petrolera venezolana se reflejó en la reducción o postergación del financiamiento a proyectos regionales del ALBA-TCP.

También el factor político ha influido en el ALBA-TCP, lo que la ha llevado a la actual etapa de resistencia. La voluntad política que condujo a varios gobiernos populares a compartir valores y una racionalidad distanciada de la dominante a partir de la emergencia del ALBA-TCP, se ve fragmentada. La primera manifestación del peligro advertido por 
algunos autores para el predominio del carácter político en la continuidad de la organización (de la Rosa y Javier, 2012), se puso de manifiesto en 2009 luego del golpe de estado perpetrado en Honduras, que provocó la deposición de su presidente; seguidamente se produjo la retirada de ese país del ALBA-TCP.

Posteriormente, se produjo una sensible contracción de esa entidad integracionista, debido a la pérdida de dos de sus miembros: Ecuador y Bolivia. Esos países habían mantenido una postura de defensa de las bases fundacionales del ALBA-TCP, e igualmente implementaron programas sociales y tomaron parte en proyectos y empresas grannacionales.

Las elecciones en Ecuador en 2018 que condujeron al poder a Lenin Moreno, significaron un giro en la política de ese país que, en el plano regional, se manifestó en el retiro de la asociación, así como también interrumpió unilateralmente el convenio de colaboración en la esfera de salud que el país sostenía con Cuba. Con posterioridad en 2019 se produce la salida de Bolivia tras la deposición de su presidente Evo Morales y, al igual que Ecuador, rescindió el convenio de colaboración con Cuba en materia de salud, con la retirada de los médicos que prestaban servicio en ese país.

A pesar de la contracción sufrida por el ALBA-TCP, esta se ha mantenido como proyecto integracionista regional, si bien con un discreto despliegue, en condiciones de agresividad del gobierno de EE.UU. sobre Venezuela y Cuba fundamentalmente. Hacia Venezuela esa agresividad se ha expresado de disímiles formas, unas de ellas más velada y otras menos, mediante el apoyo a las fuerzas opositoras y a gobiernos fronterizos, desestabilización del gobierno, campañas de descrédito, sanciones económicas, intentos de atentados y de invasión. Se conoce que desde 2015 el gobierno norteamericano ha adoptado más de 140 medidas restrictivas contra Venezuela (Telesurtv, 2020). Esas acciones encontraron resistencia en el ALBA-TCP con la adopción de declaraciones a favor de Venezuela y la celebración de una cumbre extraordinaria en marzo de 2015 para tratar la situación de ese país exclusivamente.

$\mathrm{Al}$ respecto, las cumbres de jefes de Estado sesionan cada año en el mes de diciembre teniendo como anfitriones a Cuba o Venezuela, así como también tienen lugar el Consejo Económico y el Consejo Políti- 
co. Igualmente se han realizado cumbres extraordinarias de donde han emanado declaraciones de apoyo a Venezuela (2015) y a Nicaragua (2019). Esas acciones sugieren una resistencia por parte del ALBA-TCP, al no renunciar a sus espacios de reflexión.

Por otra parte, en las circunstancias generadas por la pandemia COVID-19 dada su propagación a más de 180 países, provocando millones de enfermos y cientos de miles de fallecidos, esta instancia se proyecta con un criterio regional. Mientras los sistemas nacionales de salud colapsan, al evidenciar sus limitaciones para socorrer a una población carente de los recursos que les facilite el acceso a los tratamientos, el ALBA-TCP ha elaborado una estrategia en la que toman parte todos sus miembros, sobre la base de las relaciones de cooperación y de ventajas compartidas. Esa acción colectiva no desestima las acciones bilaterales emprendidas por sus miembros, especialmente Venezuela con el suministro de materiales e insumos médicos y Cuba con el envío de brigadas médicas a más de 25 países aquejados por la pandemia ${ }^{9}$.

La más reciente celebración del Consejo Presidencial del ALBA$\mathrm{TCP}^{10}$ y posteriormente el Consejo económico y el Consejo político ${ }^{11}$, que tuvieron como tema exclusivo el COVID-19, puede ser considerada una manifestación de la resistencia de esa instancia integracionista. Los intercambios se produjeron al más alto nivel y contó con la presencia de especialistas quienes presentaron análisis de una realidad que rebasa la dimensión de la salud (Bárcena, 2020). Los cónclaves que debieron realizarse en línea precisamente por la existencia de la pandemia, sentarían las bases para la elaboración de un plan de acción común para enfrentar la pandemia e incluso la etapa posterior en la que se combinan la economía, la política y lo social (Ramonet, 2020). Su celebración evidencia la validez de la concertación regional en la solución de problemas que forman parte de la realidad y afectan el desarrollo satisfactorio de los países y las asociaciones integracionistas.

Además de los países miembro del ALBA-TCP, tomaron parte en la reunión del Consejo presidencial, cuatro invitados: la secretaria de la

9 Antes de que se declarara la pandemia de COVID-19, 59 países contaban con la presencia de médicos cubanos, posteriormente se agregaron más de 24 países para recibir esos servicios.

10 El Consejo presidencial del ALBA-TCP tuvo lugar el 10 de junio de 2020.

11 El Consejo económico y el Consejo político se celebraron el 29 de junio de 2020. 
CEPAL, Alicia Bárcena, el expresidente boliviano Evo Morales, el director ejecutivo del Instituto Tricontinental de investigaciones sociales, Vijay Prashad, y el académico Ignacio Ramonet. Cada uno de los participantes ofreció reflexiones y propuestas, algunas de ellas de carácter global, mientras otras concernientes a la organización regional, razón por la cual fueron incluidas en la Declaración Conjunta del Consejo económico y el Consejo político celebrado días después de la Cumbre presidencial.

Durante a celebración del Consejo presidencial, además de las propuestas dirigidas al enfrentamiento a la pandemia, recibieron atención temas propios del funcionamiento y desarrollo del ALBA-TCP. Así, el fortalecimiento de la economía mediante la producción de bienes y servicios, la reactivación de la grannacional Petrocaribe y del Banco del Alba con el empleo de la moneda de cuenta sucre y del petro como moneda de cambio, centraron las intervenciones en torno al futuro del ALBA-TCP (Capote, 2020).

En el Consejo presidencial se hizo énfasis en que las medidas de enfrentamiento del COVID-19 tuvieran un carácter regional, tanto en la elaboración como en su posterior disfrute. En ese sentido, el Consejo económico y el Consejo político acordaron:

- realizar asesoramiento epidemiológico,

- atender las prioridades de los países caribeños,

- compartir y generar capacidades para el diagnóstico, y

- retomar la implementación de un sistema único para la fabricación, el registro, la regulación y los permisos de distribución de medicamentos.

Usualmente en los documentos derivados de las reuniones de alto nivel del ALBA-TCP, se incluye un acuerdo contra las medidas coercitivas hacia los países miembros de la asociación que, en esta ocasión también fue adoptado. Como el propósito del cónclave estuvo centrado en la pandemia, a la integración solo se hizo una referencia en términos de reimpulsar (Granma, 2020).

Puesto que la propuesta del ALBA-TCP está centrada en la dimensión en la que más ha avanzado, esto es, el aspecto social, ello favorece sus posibilidades de éxito, lo que concierne específicamente al 
sector de la salud, donde existen experiencias en la atención primaria y especializada, la producción de medicamentos y la formación profesional.

Una acción de ese orden no había sido emprendida con anterioridad por otro esquema en la región. Si bien se observa en el ALBA-TCP un repliegue desde el año 2015, en medio de una crisis en la que se integran las dimensiones sanitaria, económica y político social (Ramonet, 2020), la organización presenta una propuesta que es expresión de sus valores fundacionales, lo cual guarda correspondencia con las teorías derivadas de los estudios de las prácticas de resistencia, que postulan la emergencia de un proyecto de cambio como resultado.

\section{Conclusiones}

En varios esquemas integracionistas se produce la retirada de algunos de sus miembros, sus proyectos se estancan, así como también sus bases fundacionales son modificadas mediante el retraimiento del rol del Estado y el desmantelamiento de las políticas sociales.

A través de la presente investigación se pudo apreciar un cambio de escenario en la evolución del ALBA-TCP, que permite establecer dos etapas con diferencias visibles. La primera, desde su fundación hasta el año 2015 fue de avances sostenidos, caracterizada por la adhesión de miembros, la adopción de programas de cooperación y resultados de impacto en la dimensión social, así como también por sus proyectos de carácter económico.

La segunda etapa, a partir de 2015 a la fecha, se ha caracterizado por una contracción en el financiamiento de los diversos programas de desarrollo, entre ellos de los sociales y la grannacional. En esa etapa se produjo la pérdida de los líderes fundacionales del proyecto integracionista, el repliegue de la izquierda, el aumento de la agresividad del gobierno de EE.UU. y la salida de Ecuador y Bolivia, circunstancias que provocan un repliegue en el desarrollo del ALBA-TCP.

Sin embargo, cuando algunos esquemas renuncian a sus postulados primigenios, el ALBA-TCP ha continuado protegiendo sus valores fundacionales y, aunque debilitado, mantiene sus proyectos. El análisis realizado, apoyado en la resistencia como referente teórico, permite inferir que el ALBA-TCP no se encuentra ni detenido ni en retroceso, 
sino en un estado de resistencia que supone el análisis crítico de la realidad por parte de los actores, para ofrecer una nueva propuesta.

Se trata de una resistencia colectiva que actúa en el escenario internacional cuyo centro se halla en la integración económica regional y se manifiesta en la continuidad de algunos de sus proyectos, en la celebración de sus cumbres y en su presencia activa en los escenarios internacionales en los que expresa su carácter contrahegemónico.

La exploración realizada permitió interpretar la etapa por la que transita el ALBA-TCP a través de las teorías derivadas de los estudios de las prácticas de la resistencia, caracterizada por la pérdida de miembros y cierto retroceso en algunos de sus proyectos, al tiempo que ha mantenido su funcionamiento y formula propuestas en el adverso escenario al cual se adiciona la pandemia del COVID-19.

Al concluir el análisis puede afirmarse la utilidad de las teorías de la resistencia en el análisis de procesos que rebasan el nivel nacional, para situarlo en el regional donde actúa el ALBA-TCP, escenario en el cual también se manifiestan modalidades de hegemonía. Igualmente se confirma que desde la resistencia es posible elaborar nuevas propuestas frente a las prácticas dominantes en la integración económica regional y, simultáneamente, defender las bases ideológicas de la organización, propósito en el cual se pueden esperar nuevas enseñanzas del ALBA-TCP.

\section{Referencias}

Angelilli, D. (2016). Por un nuevo modelo de cooperación y desarrollo solidario y complementario. Entrevista a Luis Vasapollo. En L. Vasapollo e I. Monal, Con Gramsci en el Alba de Nuestra América (pp. 311-332). La Habana: Editorial de Ciencias Sociales.

Aponte, M. (2014). El nuevo regionalismo estratégico. Los primeros diez años del ALBA-TCP. Buenos Aires: Consejo Latinoamericano de Ciencias Sociales, CLACSO.

Aponte, M. (2018). El ALBA-TCP. Entre los regionalismos y la geopolítica. Voces en el fénix, 69, 112-119. Recuperado de https:// www.vocesenelfenix.com/content/el-alba-tcp-entre-losregionalismos-y-la-geopol\%C3\%ADtica 
Bárcena, A. (2015). El pensamiento económico en la CEPAL: pasado y presente. En A. Bárcena (Ed.), Reflexiones sobre el desarrollo en América Latina y el Caribe (pp. 51-71). Santiago de Chile: Comisión Económica para América Latina y el Caribe, CEPAL.

Bárcena, A. (2020). América Latina y el Caribe ante la pandemia del Covid-19. Efectos económicos y sociales. Santiago de Chile: Comisión Económica para América Latina y el Caribe, CEPAL.

Benzi, D. (2014). El exitoso ocaso del ALBA. Réquiem para el último vals tercermundista. Nueva Sociedad, 261. Recuperado de https://nuso.org/articulo/el-exitoso-ocaso-del-alba/

Borón, A. (2008). ALBA y TCP: posibilidades y perspectivas. En O. Martínez (Ed.), La integración en América Latina: de la retórica a la realidad (pp. 107-114). La Habana: Editorial de Ciencias Sociales.

Briceño, J. (2014). El ALBA: una discusión de su modelo, sus resultados y sus perspectivas. Anuario de integración, 10, 151178. Recuperado de http://www.cries.org/wp-content/ uploads/2014/11/6-Briceno.pdf

Chávez, H. (2005). Alba, amanecer de una nueva era. La Habana: Consejo de Estado.

Cole, K. (2010). Jazz in the Time of Globalisation: The Bolivarian Alliance for the Peoples of Our America. Third Worl Quarterly, 31(2). https://doi.org/10.1080/01436591003712015

de la Rosa, U. y Javier, F. (2012). La Alianza Bolivariana para las Américas-Tratado de Comercio de los Pueblos (ALBA-TCP): análisis de un proyecto de integración regional latinoamericana con una fuerte dimensión altermundista. Estudios Políticos, 9(25), 131-170. Recuperado de https://www.redalyc.org/ pdf/4264/426439546007.pdf

Giroux, H. (1983). Teorías de la reproducción y la resistencia en la nueva sociología de la educación: un análisis crítico. Harvard Education Review, 3. Recuperado de http://files.doctorado-en-educacion-2-cohorte.webnode.es/20000007937133380 5/teorias\%20de\%20la\%20reproduccion\%20y\%20 la\%20resistencia_Giroux.pdf

González, S., Colmenares, J., y Ramírez, V. (2011). La resistencia social: una resistencia por la paz. Hallazgos, 8(15), 237-254. Recuperado de https://www.redalyc.org/ pdf/4138/413835204013.pdf 
Granma (29 de junio de 2020). Redacción digital. Recuperado de http://www.granma.cu

Gratius, S. y Puente, J. (2018). ¿Fin del proyecto alternativo Alba? Una perspectiva política y económica. Revista de Estudios Políticos, 180, 229-252. https://doi.org/10.18042/cepc/rep.180.08

Guerrero, P. (2005). Estudio de las resistencias de los profesores a una estrategia para el desarrollo de la creatividad en tres unidades educacionales. Psykhe, 14(1), 31-45. http://dx.doi. org/10.4067/S0718-22282005000100003 Hidalgo, M. (2014).

Michel Foucault y la crítica del presente: prácticas de resistencia y transformación. Pueblos, Revista de Información y Debate, 63. Recuperado de http://www.revistapueblos.org/?p=18178

Muhr, T. (2010). Venezuela e ALBA: regionalismo contrahegemónico e ensino superior para todos. Educação e Pesquisa, 36(2), 611627. Recuperado de https://www.scielo.br/pdf/ep/v36n2/ a13v36n2.pdf

Pérez, M. (2016). Aproximación al pensamiento de Hugo Chávez: la integración económica regional. En V. Rodríguez y J. A. Castellanos (Eds.), Movimientos sociales (pp. 761-770). La Habana: Instituto de Historia de Cuba, Universidad de Chapingo.

Ramonet, I. (junio, 2020). Situación económica post pandemia. Ponencia presentada en la Conferencia sobre economía, finanzas y comercio del Alba en el marco de la COVID-19. La Habana (Ciudad). Recuperado de http//www.google.com/amp/s/ www.telesurtv.net/amp/news/venezuela-albatpc-cumbre-virtual-economia-pospandemia-20200610-0041.html xxxxxxx

Samuha, J. A. (2015). Regionalismo e integración en América Latina: balance y perspectivas. Madrid: Ministerio de Asuntos Exteriores.

Sánchez, A. (2013). Prácticas de resistencia y alternativas para el cambio. Una defensa del trabajo social con colectivos y comunidades. Investigaciones de Intervención Social, 3(4), 157176. Recuperado de https://dialnet.unirioja.es/servlet/ articulo? codigo $=5304698$

Silva, C., Noyola, A., y Kan, J. (2017). América Latina: una integración regional fragmentada y sin rumbo. Buenos Aires: Consejo Latinoamericano de Ciencias Sociales, CLACSO. 
Soto, W. y Morales, M. (2014). Centroamérica y la Alianza del Pacífico: los tratados de libre comercio con México y Perú. En W. A. Soto Acosta (Ed.), Política internacional e integración regional comparada en América Latina (pp. 233-256). San José de Costa Rica: Universidad Nacional de Costa Rica.

Stédile, J., Piñero, J., y Bertoldi, M. (2013.). La integración desde los pueblos. En I. León (Ed.), La ALBA: horizonte latinoamericano del Siglo XXI (pp. 131-140). Quito: Fundación de Estudios, Acción y Participación Social, FEDAEPS-Agencia Latinoamericana de Información, ALAI.

Telesurtv (2020). Medidas coercitivas de EE.UU. contra Venezuela. Recuperado de https://www.telesurtv.net/news/venezuelacronologia-medidas-sanciones-eeuu-20200213-0004.html

Thompson, J. B. (1998). Ideología y cultura moderna. México, D.F.: Universidad Autónoma Metropolitana-Xochimilco.

Vargas, J. (2014). A propósito de la resistencia como propuesta teórica del estudio histórico. Tiempo y espacio, 28, 7-22. Recuperado de http://www.ubiobio.cl/miweb/webfile/media/222/ Tiempo/2012/\%2328.01.pdf 